\title{
UNIVERSAL SOLUTIONS AND UNIVERSAL RELATIONS FOR SOME TYPES OF ELASTIC FLUID CRYSTALS*
}

BY

\author{
C. -C. WANG \\ Rice University
}

1. Introduction. Universal solutions and universal relations for a fixed type ${ }^{1}$ of materials are important results in the theory of elasticity. Until recently, results belonging to those two categories concerned almost exclusively isotropic solids, fluids, and some types of solid crystals. In this paper, I present some universal solutions and universal relations for two types of simple fluid crystals, called subfluids, which I introduced in my earlier paper [1].

Specifically, I consider in this paper the type of subfluids possessing one preferred line and the type of subfluids possessing one preferred plane. Let $\left\{\mathbf{e}_{1}, \mathbf{e}_{2}, \mathbf{e}_{3}\right\}$ be an orthonormal basis in a reference configuration such that $e_{3}$ is in the direction of the preferred line, and let $\left\{\mathbf{e}_{1}, \mathbf{e}_{2}\right\}$ span the preferred plane. Then the component matrix of a tensor $\mathbf{P}$ belonging to the isotropy group of the first type is

$$
\left[P_{i i}\right]=\left[\begin{array}{lll}
a & b & 0 \\
c & d & 0 \\
e & f & g
\end{array}\right],
$$

while that of the second type is

$$
\left[P_{i i}\right]=\left[\begin{array}{ccc}
a & b & c \\
d & e & f \\
0 & 0 & g
\end{array}\right],
$$

where for both types $a, \cdots, g$ are arbitrary real numbers such that

$$
\left|\operatorname{det}\left[P_{i j}\right]\right|=1 \text {. }
$$

In a deformed configuration with deformation gradient $\mathrm{F}$ relative to the reference configuration, the preferred line, of course, is in the direction of the unit vector

$$
\mathrm{i}=\mathrm{Fe}_{3} /\left|\mathrm{Fe}_{3}\right| \text {, }
$$

and the preferred plane is spanned by the set $\left\{\mathrm{Fe}_{1}, \mathrm{Fe}_{2}\right\}$, so that unit normal of the preferred plane is

$$
\mathrm{n}=\mathrm{Fe}_{1} \times \mathrm{Fe}_{2} /\left|\mathrm{Fe}_{1} \times \mathrm{Fe}_{2}\right|
$$

${ }^{*}$ Received February 15, 1969; revised version received March 20, 1969.

${ }^{1}$ We use the term type strictly in the following sense: A type of materials is a class of materials possessing a fixed material symmetry; the material symmetry is characterized by the isotropy group of the constitutive relation. 
In my paper [1], I proved that the stress tensor $\mathrm{T}$ in the deformed configuration for the two types of subfluids is given by

$$
\mathbf{T}=f_{0}(\rho) \mathbf{I}+f_{1}(\rho) \mathbf{i} \otimes \mathbf{i},
$$

and

$$
\mathbf{T}=f_{0}(\rho) \mathbf{I}+f_{1}(\rho) \mathbf{n} \otimes \mathbf{n},
$$

respectively, where $f_{0}$ and $f_{1}$ are functions of the density $\rho$ provided that the materials are not subjected to any internal constraint nor required to have a stored-energy function.

Now suppose that the subfluids are hyper-elastic, so that they possess a stored-energy function. Then I proved in my paper [1] that the constitutive equations become

$$
\mathbf{T}=f_{0}(\rho) \mathbf{I}+f_{\rho} \mathbf{i} \otimes \mathbf{i},
$$

and

$$
\mathbf{T}=f_{0}(\rho) \mathbf{I}+f \rho \mathbf{n} \otimes \mathbf{n},
$$

respectively, where $f_{0}$ is a function of $\rho$ and where $f$ is a (material) constant.

Finally, suppose that the subfluids are incompressible. Then the constitutive equations are

$$
\mathbf{T}=-p \mathbf{I}+f \mathbf{i} \otimes \mathbf{i},
$$

and

$$
\mathbf{T}=-p \mathbf{I}+f \mathbf{n} \otimes \mathbf{n},
$$

respectively, where $p$ is an undetermined hydrostatic stress, and where $f$ is a (material) constant. In this case, the deformation gradient must satisfy the incompressibility condition:

$$
|\operatorname{det} \mathbf{F}|=1 \text {, }
$$

where $\operatorname{det} \mathbf{F}$ denotes the determinant of $\mathbf{F}$.

Some universal solutions and universal relations based on the constitutive equations (1.6)-(1.11) are obtained in this paper. Specifically, statical universal solutions for the compressible subfluids are considered in Sec. 2; an example of the statical universal solutions, for which a complete set of universal relations is worked out, is treated in Sec. 3. Statical universal solutions for the incompressible subfluids are considered in Sec. 4, and finally, some dynamical universal solutions are given in Sec. 5 .

2. Statical universal solutions (for the compressible subfluids). Since the constitutive equations (1.6) and (1.7) are formally the same, we can analyze the field equations for the two types of subfluids within one scheme. For definiteness, we denote the constitutive equation as

$$
\mathbf{T}=f_{0}(\rho) \mathbf{I}+f_{1}(\rho) \mathbf{e} \otimes \mathbf{e},
$$

and we assume that the reference configuration is a homogeneous configuration, so that $f_{0}$ and $f_{1}$ do not depend on the material particle. Further, we assume that no body force is acting on the subfluid. Then the equation of equilibrium is

$$
\operatorname{div} \mathbf{T}=\mathbf{0} .
$$


Substituting $\mathbf{T}$ from (2.1) into this equation, we obtain

$$
\operatorname{grad} f_{0}(\rho)+\operatorname{div}\left(f_{1}(\rho) \mathbf{e} \otimes \mathbf{e}\right)=\mathbf{0},
$$

which can be expressed by the chain rule as

$$
\frac{d f_{0}}{d \rho} \operatorname{grad} \rho+\frac{d f_{1}}{d \rho}(\mathbf{e} \cdot \operatorname{grad} \rho) \mathbf{e}+f_{1}\{[\operatorname{grad} \mathbf{e}] \mathbf{e}+\mathbf{e}(\operatorname{div} \mathbf{e})\}=\mathbf{0} .
$$

Since we seek universal solutions for a whole type of subfluids, the functions $f_{0}$ and $f_{1}$ are arbitrary. Consequently, equation (2.4) is satisfied if and only if

$$
\operatorname{grad} \rho=0,
$$

and

$$
[\operatorname{grad} \mathbf{e}] \mathrm{e}+\mathrm{e}(\operatorname{div} \mathbf{e})=\mathbf{0} .
$$

We pause to remark that (2.5) and (2.6) are also the governing equations for the universal solutions of the hyperelastic subfluids. This fact is more or less obvious. Had we used the constitutive equation

$$
\mathbf{T}=f_{0}(\rho) \mathbf{I}+f_{\rho} \mathbf{e} \otimes \mathbf{e}
$$

for the hyperelastic subfluids, we would obtain, instead of (2.4), the equation

$$
\frac{d f_{0}}{d \rho} \operatorname{grad} \rho+f(\mathbf{e} \cdot \operatorname{grad} \rho) \mathrm{e}+f \rho\{[\operatorname{grad} \mathrm{e}] \mathrm{e}+\mathrm{e}(\operatorname{div} \mathrm{e})\}=\mathbf{0} .
$$

But since $f_{0}$ and $f$ are arbitrary, a necessary and sufficient condition for (2.8) is again the system (2.5) and (2.6). Thus we have proved that the universal solutions for the elastic subfluids coincide with those for the hyperelastic subfluids.

Now we consider the governing equations (2.5) and (2.6). Equation (2.5), of course, has the trivial solution:

$$
\rho=\text { constant }
$$

or equivalently

$$
|\operatorname{det} \mathbf{F}|=\text { constant. }
$$

The crucial governing condition for a universal solution is equation (2.6), together with the intrinsic restriction that $\mathrm{e}$ is a unit vector,

$$
\mathrm{e} \cdot \mathrm{e}=1 .
$$

Taking the gradient of (2.11), we obtain

$$
[\operatorname{grad} \mathrm{e}]^{T} \mathrm{e}=\mathbf{0},
$$

where the superscript $T$ denotes the transposition. This equation implies that the quadratic form of the tensor [grad e] vanishes at $\mathbf{e}$,

$$
\mathrm{e} \cdot[\operatorname{grad} \mathrm{e}] \mathrm{e}=0 .
$$

Now taking the dot product of (2.6) with e, and using (2.11) and (2.13), we obtain

$$
\operatorname{div} \mathrm{e}=0 .
$$

Thus e must be a solenoidal field. 
We can substitute (2.14) into (2.6), obtaining

$$
[\operatorname{grad} e] \mathrm{e}=\mathbf{0} .
$$

This equation means that the value of e does not change along any integral curve of the vector field $\mathbf{e}$, so that the integral curves of $\mathbf{e}$ are all straight lines.

Finally, combining (2.12) and (2.15), we see that

$$
\mathrm{e} \times \operatorname{curl} \mathrm{e}=\mathbf{0} \text {. }
$$

Thus e is a screw field provided that curl e does not vanish.

We now consider the two types of subfluids separately.

Case 1. $\mathbf{e}=\mathbf{n}$.

In this case the vector field $\mathbf{n}$ satisfies one more restriction:

$$
\mathrm{n} \cdot \operatorname{curl} \mathrm{n}=0,
$$

since $\mathbf{n}$ is the normal field of a family of surfaces-which are the deformed configurations of the parallel preferred planes. Combining (2.17) and (2.16), and using the fact that n does not vanish, we obtain

$$
\operatorname{curl} \mathbf{n}=\mathbf{0}
$$

Thus $\mathbf{n}$ is a lamellar field.

Now taking the gradient of (2.12), and using the condition (2.14), we obtain

$$
\operatorname{tr}\left([\operatorname{grad} n]^{2}\right)=0,
$$

where tr denotes the trace. But from (2.18) we have also

$$
\operatorname{tr}\left\{\left([\operatorname{grad} \mathbf{n}]-[\operatorname{grad} \mathbf{n}]^{T}\right)^{2}\right\}=0 .
$$

Substituting (2.19) into (2.20), we obtain

$$
\operatorname{tr}\left([\operatorname{grad} \mathbf{n}][\operatorname{grad} \mathbf{n}]^{T}\right)=0,
$$

which means that

$$
\operatorname{grad} \mathbf{n}=\mathbf{0} .
$$

Consequently, $\mathbf{n}$ is a constant vector in the deformed configuration. This result means that the deformed configuration is also a homogeneous configuration.

The deformation from one homogeneous configuration onto another one, however, need not be a homogeneous deformation. For example, any shear deformation preserving the preferred planes in the reference configuration is a universal solution.

Case 2. $\mathbf{e}=\mathbf{i}$.

In this case the vector field $\mathrm{i}$ need not be lamellar or complex lamellar. The governing equations for $\mathbf{i}$ are

$$
\begin{gathered}
\mathbf{i} \cdot \mathbf{i}=1, \\
\operatorname{div} \mathbf{i}=0,
\end{gathered}
$$

and

$$
\mathbf{i} \times \operatorname{curl} \mathbf{i}=\mathbf{0} \text {. }
$$


A general solution for this system has not been found. Under condition (2.23), a necessary and sufficient condition for (2.25) is that the integral curves of $\mathbf{i}$ be straight lines. This result is a theorem of Neményi and Prim [2], the necessity part of which we have proved in (2.15). Under condition (2.25), a necessary and sufficient condition for (2.24) is

$$
\mathbf{i} \cdot \operatorname{grad}(|\operatorname{curl} \mathbf{i}|)=0 \text {, }
$$

which means that the surfaces of constant abnormality are vector sheets of $i$. This result is a theorem of Ballabh [3]. Combining these two results, we see that the surfaces

$$
\mid \text { curl } \mathrm{i} \mid=\text { constant }
$$

are ruled surfaces.

We can visualize the vector field $i$ as the velocity field of a steady flow. Then condition (2.23) says that the flow is of constant and uniform speed, condition (2.24) says that the flow is incompressible, and finally, the theorem of Neményi and Prim says that the streamlines of the flow are straight lines. Intuitively, we know that besides the uniform solution obtained in the previous case, there is a family of nonuniform solutions having the components

$$
i^{1}=0, \quad i^{2}=\cos \theta\left(x^{1}\right), \quad i^{3}=\sin \theta\left(x^{1}\right)
$$

relative to a rectangular Cartesian coordinate system $\left(x^{1}, x^{2}, x^{3}\right)$. Here the angle $\theta$ is an arbitrary function of $x^{1}$. For this family of solutions, the surfaces of constant abnormality are the coordinate planes perpendicular to the $x^{1}$-axis, and the integral curves of $\mathbf{i}$ are parallel straight lines on each of those parallel coordinate planes.

It is not known whether or not any further solutions for the system (2.23)-(2.25) exist.

3. Universal relations in simple shears. A simple shear is a homogeneous deformation whose deformation gradient has the component matrix

$$
\left[F_{i j}\right]=\left[\begin{array}{lll}
1 & 0 & 0 \\
k & 1 & 0 \\
0 & 0 & 1
\end{array}\right]
$$

relative to an orthonormal basis $\left\{\mathbf{e}_{1}, \mathbf{e}_{2}, \mathbf{e}_{3}\right\} ; k$ is an arbitrary real number, called the amount of shear. We consider the following two cases:

Case 1. Subfluids whose preferred plane is spanned by $\left\{\mathbf{e}_{1}, \mathbf{e}_{3}\right\}$ in the reference configuration.

In this case the unit normal $\mathbf{n}$ in the deformed configuration has the components

$$
n^{1}=\sin \theta, \quad n^{2}=-\cos \theta, \quad n^{3}=0,
$$

where $\theta$ is the angle of shear given by

$$
\tan \theta=k .
$$

Substituting (3.2) into (1.7), we see that the stress tensor has the components

$$
\begin{aligned}
& T_{11}=F_{0}+F_{1} \sin ^{2} \theta, \\
& T_{22}=F_{0}+F_{1} \cos ^{2} \theta,
\end{aligned}
$$




$$
\begin{aligned}
& T_{33}^{-\cdots}=F_{0}, \\
& T_{12}=-F_{1} \sin \theta \cos \theta,
\end{aligned}
$$

and

$$
T_{13}=T_{23}=0,
$$

where $F_{0}$ and $F_{1}$ are the values of $f_{0}$ and $f_{1}$ in the reference configuration. From (3.4), we have the following universal relations:

$$
\frac{T_{11}-T_{33}}{T_{12}}=k
$$

and

$$
\frac{T_{22}-T_{33}}{T_{12}}=-\frac{1}{k}
$$

We claim that these are the only nontrivial universal relations in this deformation. Specifically, we have the following

Proposition. A symmetric tensor $\mathbf{K}$ has the form

$$
\mathrm{K}=K_{0} \mathbf{I}+K_{1} \mathbf{n} \otimes \mathbf{n}
$$

if and only if the components of $\mathbf{K}$ relative to $\left\{\mathbf{e}_{1}, \mathbf{e}_{2}, \mathbf{e}_{3}\right\}$ satisfy the conditions

$$
\begin{aligned}
& K_{13}=K_{23}=0, \\
& \frac{K_{11}-K_{33}}{K_{12}}=-k,
\end{aligned}
$$

and

$$
\frac{K_{22}-K_{33}}{K_{12}}=-\frac{1}{k}
$$

Further, if these conditions are satisfied, then

$$
K_{0}=K_{33}
$$

and

$$
K_{1}=K_{11}+K_{22}-2 K_{33} .
$$

Thus $K_{0}$ and $K_{1}$ are constants if and only if

$$
K_{33}=\text { constant }
$$

and

$$
K_{11}+K_{22}=\text { constant. }
$$

The proof of this proposition is obvious. We have shown the necessity part in $(3.4)_{5}$, (3.5), and (3.6). Conversely, if (3.8)-(3.10) hold, then it can be shown easily that $\mathbf{n}$ and any vector perpendicular to $\mathrm{n}$ are proper vectors of $\mathbf{K}$. Thus $\mathrm{K}$ has the form (3.7). The conditions (3.11) and (3.12) are direct consequences of (3.4). 
From this proposition, we see that $(3.4)_{5},(3.5),(3.6)$, and the conditions

$$
T_{33}=\text { constant }
$$

and

$$
T_{11}+T_{22}=\text { constant }
$$

form a complete set of universal relations in this case, since the form

$$
\mathbf{T}=F_{0} \mathbf{I}+F_{1} \mathbf{n} \otimes \mathbf{n}
$$

gives completely the restriction on the stress tensor due to the material symmetry.

Case 2. Subfluids whose preferred line is spanned by $\mathbf{e}_{2}$ in the reference configuration. In this case the unit vector $\mathbf{i}$ in the deformed configuration has the components

$$
i^{1}=\cos \theta, \quad i^{2}=\sin \theta, \quad i^{3}=0 .
$$

Substituting (3.18) into (1.6), we obtain

$$
\begin{aligned}
& T_{11}=F_{0}+F_{1} \cos ^{2} \theta, \\
& T_{22}=F_{0}+F_{1} \sin ^{2} \theta, \\
& T_{33}=F_{0}, \\
& T_{12}=F_{1} \sin \theta \cos \theta .
\end{aligned}
$$

and

$$
T_{13}=T_{23}=0 .
$$

By the same argument as before, we see that a complete set of universal relations for this case is $(3.19)_{5},(3.15),(3.16)$, together with

$$
\frac{T_{11}-T_{33}}{T_{12}}=\frac{1}{k}
$$

and

$$
\frac{T_{22}-T_{33}}{T_{12}}=k .
$$

Remark. Since simple shears constitute only a special class of deformations, the behavior of a material in simple shear does not determine the response function completely. Thus universal relations in simple shear are necessary but not sufficient conditions for a fixed type of material. There may be many other types of materials whose complete sets of universal relations in simple shears are the same as those for Case $\mathbf{1}$ or those for Case 2.

4. Statical universal solutions (for incompressible subfluids). As before, we write the constitutive equations (1.10) and (1.11) for the two types of subfluids in a common form

$$
\mathbf{T}=-p \mathbf{I}+f \mathbf{e} \otimes \mathbf{e},
$$

where $p$ is an undetermined pressure, and where $f$ is a constant. Substituting (4.1) into the equation of equilibrium (2.2), we obtain

$$
f \operatorname{div}(\mathrm{e} \otimes \mathrm{e})=\operatorname{grad} p .
$$


Now since we seek universal solutions, the constant $f$ in this equation is arbitrary. Hence the governing equation for $\mathbf{e}$ is

$$
\operatorname{curl}(\operatorname{div}(\mathbf{e} \otimes \mathbf{e}))=\mathbf{0} .
$$

Of course, e must satisfy condition (2.11) also.

A general solution for the system (4.3) and (2.11) has not been found. Besides the previous uniform solution (2.22) and the particular solution (2.28) for the compressible subfluids, we can exhibit three new families of nonuniform solutions. Again we consider the following two cases:

Case $1 . \quad \mathbf{e}=\mathbf{i}$. The solutions are

Family 1. The physical components of i relative to a cylindrical coordinate system $(r, \theta, z)$ have the form

$$
i\langle r\rangle=\cos \alpha, \quad i\langle\theta\rangle=\sin \alpha, \quad i\langle z\rangle=0,
$$

where $\alpha$ is a constant angle. The pressure field for this family is given by

$$
p=f(\cos 2 \alpha \log \gamma+\theta \sin 2 \alpha)+p_{0},
$$

where $p_{0}$ is an arbitrary constant.

Two special cases of (4.4) are

(I) Plane radial distribution, with $\alpha=0$.

$$
i\langle r\rangle=1, \quad i\langle\theta\rangle=i\langle z\rangle=0 .
$$

This field is lamellar but is not solenoidal.

(II) Plane circular distribution, with $\alpha=\pi / 2$.

$$
i\langle r\rangle=i\langle z\rangle=0, \quad i\langle\theta\rangle=1 .
$$

This field is complex-lamellar and solenoidal but is not a screw field.

Family 2. The physical components of $\mathrm{i}$ relative to a spherical coordinate system $(r, \theta, \varphi)$ have the form

$$
i\langle r\rangle=1, \quad i\langle\theta\rangle=i\langle\varphi\rangle=0 .
$$

This field forms a central radial distribution, which is lamellar but is not solenoidal.

The pressure field for this family is given by

$$
p=2 f \log r+p_{0}
$$

where $p_{0}$ is a constant.

Family 3. The physical components of $\mathrm{i}$ relative to a cylindrical coordinate system $(r, \theta, z)$ have the form

$$
i\langle r\rangle=0, \quad i\langle\theta\rangle=\cos \alpha(r), \quad i\langle z\rangle=\sin \alpha(r),
$$

where $\alpha(r)$ is an arbitrary function of $r$. This field is solenoidal but is not a screw field.

The pressure field for this family is given by

$$
p=-f \int_{r_{0}}^{r} \frac{\cos ^{2} \alpha(r)}{r} d r+p_{0}
$$


where $p_{0}$ is a constant. The plane radial distribution (4.7) is also a special case of this family, with $\alpha(r)$ equal to zero.

Case 2. $\mathrm{e}=\mathrm{n}$. In this case restriction (2.17) must also be satisfied. The reader will verify easily that the first two families in the previous case satisfy this additional condition, and hence they remain universal solutions with $\mathbf{i}$ replaced by $\mathrm{n}$. The third family, however, does not satisfy (2.17) in general. Those belonging to Family 3 and satisfying (2.17) are given by the following

Family $3^{\prime}$. The physical components of $\mathbf{n}$ relative to a cylindrical coordinate system $(r, \theta, z)$ have the form

$$
n\langle r\rangle=0, \quad n\langle\theta\rangle=\cos \alpha(r), \quad n\langle z\rangle=\sin \alpha(r),
$$

with

$$
\sin 2 \alpha(r)=\alpha_{0} / r^{2},
$$

where $\alpha_{0}$ is a constant. To prove this we compute the components of the curl on $\mathbf{n}$ :

$$
\begin{aligned}
& (\operatorname{curl} n)\langle r\rangle=0, \\
& (\operatorname{curl} n)\langle\theta\rangle=-\alpha^{\prime} \cos \alpha,
\end{aligned}
$$

and

$$
(\operatorname{curl} n)\langle z\rangle=\left(\cos \alpha-r \alpha^{\prime} \sin \alpha\right) r^{-1} .
$$

Substituting (4.12) and (4.14) into (2.17), we see that $\alpha$ must satisfy the condition

$$
2 r \alpha^{\prime} \cos 2 \alpha+\sin 2 \alpha=0 .
$$

(4.13) is an integral of this equation.

Note. In order that the angle $\alpha$ be real, $r^{2}$ must be greater than the absolute value of the constant $\alpha_{0}$. When $\alpha_{0}$ is equal to zero, the solution (4.12) reduces to the plane circular distribution with $\alpha=0$, or to the uniform field with $\alpha=\pi / 2$.

5. Dynamical universal solutions (for the incompressible subfluids). For incompressible elastic bodies, Truesdell [4] has considered a class of exact dynamical solutions satisfying the following condition: At each time $t$, the instantaneous configuration is a possible configuration of static equilibrium under surface forces exerted on the boundary of the body. Such solutions are called quasi-equilibrated motions. In general, a quasiequilibrated motion is dynamically possible if and only if the acceleration field is a conservative field with a single-valued acceleration potential $\zeta$, viz.

$$
\mathrm{a}=\operatorname{grad} \zeta \text {. }
$$

Then the dynamical pressure field $p$ is related to the static equilibrium pressure field $P$ by

$$
p=P-\rho \zeta,
$$

where $\rho$ denotes the density.

We proceed to set forth some families of quasi-equilibrated motions for the two types of incompressible subfluids. Again we write the constitutive equation in general as (4.1). Then we consider

Case 1. $\mathrm{e}=\mathrm{i}$. 
We consider the following families of motions:

Family 1. Flow between coaxial circular cylinders.

The deformation functions are

$$
r=R, \quad \theta=\Theta+f(R, t), \quad z=Z+g(R, t),
$$

where $f(R, t)$ and $g(R, t)$ are two unknown functions whose forms must be determined by the condition (5.1). For this family, we assume that the initial distribution of the preferred axis $\mathbf{i}$ is given by (4.10). From (5.3), the physical components of the deformation gradient $\mathbf{F}$ are given by

$$
[F\langle i A\rangle]=\left[\begin{array}{ccc}
1 & 0 & 0 \\
R \partial f / \partial R & 1 & 0 \\
\partial g / \partial R & 0 & 1
\end{array}\right] .
$$

Then

$$
\operatorname{det} \mathbf{F}=1
$$

and

$$
\mathbf{F i}=\mathbf{i} .
$$

Thus the motion is a volume-preserving quasi-equilibrated motion.

Now we consider the restriction on the motions due to the condition (5.1). First, we determine the covariant components of the acceleration field $a$ in $(r, \theta, z)$ :

$$
a_{r}=-r f^{\prime 2}, \quad a_{\theta}=r^{2} f^{\prime \prime}, \quad a_{z}=g^{\prime \prime},
$$

where the prime denotes partial derivative with respect to the time $t$. From (5.7), condition (5.1) is satisfied if and only if

$$
\partial r^{2} f^{\prime \prime} / \partial r=0, \quad \partial g^{\prime \prime} / \partial r=0 .
$$

These two differential equations can be integrated at once, yielding the complete solution

$$
f(R, t)=A(t) / R^{2}+B(R) t+C(R)
$$

and

$$
g(R, t)=D(t)+E(R) t+F(R),
$$

where $A, \cdots, F$ are arbitrary functions. For this solution the velocity field $v$ has the physical components

$$
v\langle r\rangle=0, \quad v\langle\theta\rangle=A^{\prime}(t) / r+r B(r), \quad v\langle z\rangle=D^{\prime}\langle t\rangle+E(r) .
$$

Thus the flow is steady if and only if $A^{\prime}$ and $D^{\prime}$ are constants.

Family 2. Twisting and radial expansion flou.

The deformation functions are

$$
r=\left(A(t) R^{2}+B(t)\right)^{1 / 2}, \quad \theta=\Theta+g(R, t), \quad z=Z / A(t)+C(t),
$$

where $A, B, C$, and $g$ are arbitrary functions, with $A \neq 0$. We assume again that the 
initial distribution of the preferred axis $\mathbf{i}$ is given by (4.9). However, unlike the previous family, this family of motions does not preserve the field i. Indeed, from (5.12), the physical components of the deformation gradient $\mathbf{F}$ form the matrix

$$
[F\langle i A\rangle]=\left[\begin{array}{ccc}
A R / r & 0 & 0 \\
r \partial g / \partial R & r / R & 0 \\
0 & 0 & 1 / A
\end{array}\right] .
$$

Thus $\mathbf{F}$ preserves the form (4.9) but not the angle $\alpha$; i.e., in the motion (5.12) the preferred axis $\mathbf{i}$ has the components

$$
i\langle r\rangle=0, \quad i\langle\theta\rangle=\cos \alpha(r, t), \quad i\langle z\rangle=\sin \alpha(r, t) .
$$

Since each instantaneous configuration is a possible statical equilibrium configuration, the motion given by (5.12) is quasi-equilibrated.

Next we determine the restrictions on the functions $A, B, C$, and $g$ due to condition (5.1). From (5.12), $a_{r}$ and $a_{\theta}$ are functions of $r$ and $t$, while $a_{z}$ is a function of $z$ and $t$. Hence the restriction due to (5.1) is

$$
\partial a_{\theta} / \partial r=0 .
$$

The reader will verify easily that, in terms of $A, B, C$, and $g$, this condition takes the form

$$
\left(A R^{2}+B\right) g^{\prime \prime}+\left(A^{\prime} R^{2}+B^{\prime}\right) g^{\prime}=\text { a function of } t .
$$

The complete solution of this equation is given by

$$
A, B, C,=\text { arbitrary functions of } t \text {, with } A \neq 0 \text {, }
$$

and

$$
g(R, t)=\int_{0}^{t} \frac{D(t)+E(R)}{A(t) R^{2}+B(t)} d t+F(R),
$$

where $D, E$, and $F$ are arbitrary functions.

Family 3.

$$
r=A R, \quad \theta=B \log R+C \Theta, \quad z=D Z,
$$

where $A, \cdots, D$ are functions of $t$ such that

$$
A^{2} C D=1 .
$$

This is a known family of quasi-equilibrated motions for incompressible isotropic solids (cf. Carroll [5]). We claim that this family is quasi-equilibrated for the subfluids if the initial distribution of the preferred axis $\mathbf{i}$ is given by (4.3). This fact is more or less obvious, since the motion (5.17) preserves the form of (4.3); i.e., in the motion (5.17) the preferred axis $\mathbf{i}$ has the components

$$
i\langle r\rangle=\cos \alpha(t), \quad i\langle\theta\rangle=\sin \alpha(t), \quad i\langle z\rangle=0,
$$

where $\alpha(t)$ is a function of $t$. Since the instantaneous configurations of this family are all possible static equilibrium configurations, the motions are quasi-equilibrated for the subfluid. 
The analysis for the restriction on the functions $A, \cdots, D$ due to the condition (5.1) is, of course, the same as that for the isotropic solids, since (5.1) is a condition on the acceleration field, which is independent of the material.

Family 4.

$$
r^{3}= \pm R^{3}+A, \quad \theta= \pm \Theta, \quad \varphi=\Phi,
$$

where $A$ is a function of $t$. This is again a known family of quasi-equilibrated motions for incompressible isotropic solids (cf. Truesdell [4]). We claim that this family is quasiequilibrated for the subfluids if the initial distribution of the preferred axis $i$ is given by (4.7). This fact is again obvious, since the motion preserves the field i.

Case 2. $\mathrm{e}=\mathrm{n}$.

The previous four families of motions remain universal solutions with respect to the following distributions of $\mathbf{n}$ :

Family 1.

$$
n\langle r\rangle=1, \quad n\langle\theta\rangle=n\langle z\rangle=0 .
$$

Family 2.

$$
n\langle r\rangle=n\langle\theta\rangle=0, \quad n\langle z\rangle=1,
$$

or the distribution (5.21).

Family 3.

$$
n\langle r\rangle=\cos \alpha(t), \quad n\langle\theta\rangle=\sin \alpha(t), \quad n\langle z\rangle=0,
$$

or the distribution (5.22).

Family 4.

$$
n\langle r\rangle=1, \quad n\langle\theta\rangle=n\langle\varphi\rangle=0 .
$$

Acknowledgment. The support of this research by a grant of the U. S. National Science Foundation to Rice University is gratefully acknowledged.

\section{ReFERENCES}

[1] C. -C. Wang, A general theory of subfluids, Arch. Rational Mech. Anal. 20, 1-40 (1965)

[2] P. F. Neményi and R. C. Prim, On the steady Beltrami flow of a perfect gas, Proc. VII Internat. Congr. Appl. Mech. London, Vol. II, Part 1, 300-314 (1948)

[3] R. Ballabh, Superposable fluid motions, Proc. Benares Math. Soc. (N.S.) 2, 69-79 (1940)

[4] C. Truesdell, Solutio generalis et accurata problematum quamplurimorum de motu corporum elasticorum incomprimibilium in deformationibus valde magnis, Arch. Rat. Mech. Anal. 11, 106-113 (1962)

[5] M. M. Carroll, Controllable deformations of incompressible simple materials, Internat. J. Engrg. Sci. 5, $515-525(1967)$ 